\title{
Searches for rare and lepton-flavour-violating Higgs boson decays in ATLAS
}

\author{
Giovanni Marchiori*广 \\ LPNHE Paris \\ E-mail: giovanni.marchiorielpnhe.in2p3.fr
}

Searches are presented for rare and lepton-flavour violating decays of the Higgs boson. The searches are performed using up to $139 \mathrm{fb}^{-1}$ of $\sqrt{s}=13 \mathrm{TeV}$ proton-proton collision data collected with the ATLAS detector at the Large Hadron Collider. The final states under study are: (i) a photon and a meson or a $Z$ boson, (ii) a charm-anticharm quark pair, (iii) a $\tau$ lepton and an electron or a muon, and (iv) a dimuon pair. No significant excess with respect to the expected background is observed in the data for any of these final states, and upper limits are set on the production cross section times decay branching ratio for each of them.

European Physical Society Conference on High Energy Physics - EPS-HEP2019 -

10-17 July, 2019

Ghent, Belgium

* Speaker.

${ }^{\dagger}$ on behalf of the ATLAS Collaboration 


\section{Introduction}

The discovery of the Higgs boson in 2012 by the ATLAS and CMS Collaborations at the Large Hadron Collider (LHC) has completed the Standard Model (SM) of particle physics ${ }^{1}$, but some experimental observations (such as that of dark matter) as well as theory arguments (e.g. the hierarchy problem) may imply that the SM is just the low-energy limit of a more fundamental theory. Deviations of the Higgs boson properties with respect to the SM predictions might provide hints of this new theory.

So far, the observed Higgs boson properties are in good agreement with the SM. In particular, its couplings to the weak gauge bosons $(W, Z)$ or to the fermions of the third generation $(\tau, t, b)$ agree with the predictions within an accuracy varying between few $\%$ and $15 \%$. Rarer Higgs boson decays that are induced by couplings to the first two generations of fermions, and flavour-violating decays, are significantly less constrained experimentally, and larger deviations of their production rates (cross section times branching ratio, $\sigma \times B R$ ) from the SM predictions are possible.

In this presentation, a few recent searches are shown for rare or lepton-flavour violating decays of the Higgs bosons produced in proton-proton $(p p)$ collisions at a centre-of-mass energy $\sqrt{s}$ of $13 \mathrm{TeV}$ and recorded by the ATLAS detector [1].

\section{Analysis strategy}

All the analyses presented here perform a full reconstruction of the final state under study (except for neutrinos), apply some selection criteria to enhance the signal (S) to background (B) ratio, and then search for a localized excess - induced by the signal being looked for - in the distribution of a quantity $x$ that is able to statistically discriminate between signal and background events (e.g. the invariant mass of the final state products of the Higgs boson decay). The signal yield and its statistical significance are determined by means of a profile-likelihood-ratio method, in which the distribution of the $x$ variable in data is fit with the sum of a model describing the signal and one or more models characterising the background (either treated inclusively or split into the sum of its various components). Systematic uncertainties are incorporated in the likelihood through "constraint terms", and "profiled" by the fit.

\section{Searches for Higgs boson decays to a photon and a $Z$ boson or a meson}

The $H \rightarrow Z \gamma$ decay in the SM is induced by loop-mediated Feynman diagrams and is expected to have a branching ratio of $1.5 \times 10^{-3}$, similar to that of the well established $H \rightarrow \gamma \gamma$ decay. However, due to trigger limitations, only $Z$ boson decays to $e e$ and $\mu \mu$ final states $(B R=6.7 \%)$ can be used to efficiently select $H \rightarrow Z \gamma$ candidates. The overall SM BR for $H \rightarrow Z \gamma \rightarrow \ell \ell \gamma(\ell=e, \mu)$ is thus $10^{-4}$. Theories beyond the SM (BSM) with new particles running in the loops or predicting a composite Higgs boson can alter this prediction.

The $H \rightarrow M \gamma$ decay, where $M$ is a $q \bar{q}$ meson, is induced by the sum of a "direct" amplitude $(H \rightarrow q \bar{q} \rightarrow M+\gamma)$ and an indirect one $\left(H \rightarrow \gamma^{*} \gamma \rightarrow M+\gamma\right)$. An anomalous value of the $H q q$ Yukawa coupling $y_{q}$ can modify the size of the former amplitude and thus the total rate of this

\footnotetext{
${ }^{1}$ right-handed neutrinos should also be introduced to generate the neutrino mass terms
} 
process. The SM predictions for the BR of these decays vary between $2 \times 10^{-5}$ for $M=\rho$ (sensitive to $\left.y_{u, d}\right)$ to $1-3 \times 10^{-6}$ for $M=\phi\left(y_{s}\right), J / \psi$ and $\psi(2 S)\left(y_{c}\right)$.

ATLAS has searched for $Z \gamma$ [2] and $M \gamma\left(M=\rho, \phi\right.$ [3], $\psi, \Upsilon$ [4]) decays with 32-36 fb $\mathrm{fb}^{-1}$ of $13 \mathrm{TeV} p p$ collisions. Events are selected that contain a photon and a same-flavour oppositesign $Z$ boson or $M$ meson decay $\left(Z \rightarrow e e / \mu \mu, \rho \rightarrow \pi^{+} \pi^{-}, \phi \rightarrow K^{+} K^{-}\right.$and $\left.\psi, \Upsilon \rightarrow \mu \mu\right)$. An excess around $125 \mathrm{GeV}$ is searched for in the distribution of the invariant mass of the photon and of the two charged particles from the $M / Z$ boson. In the $H \rightarrow Z \gamma$ search, the sensitivity of the analysis is increased by classifying events in categories with different $S / B$ that are then analysed in parallel. No significant excess is found (see Figure 1(a) for an example) and $95 \%$ confidence level (CL) upper limits are set on $\sigma \times B R$ between $6.6(Z \gamma)$ and 50-200 $(M=\rho, \phi, J / \psi)$ times the SM predictions. Upper limits of 2000 times the SM prediction are set for $M=\psi(2 S)$ and $\Upsilon(n S)$, $n=1,2,3$.

\section{Search for the $H \rightarrow c \bar{c}$ decay}

The $H \rightarrow c \bar{c}$ decay provides a direct probe of the $y_{c}$ Yukawa coupling. The BR of this decay is $3 \%$ in the SM and thus a large signal yield is expected. However, an inclusive search of the process $p p \rightarrow H \rightarrow c \bar{c}$ is prohibitive due to the QCD background which is several orders of magnitude larger. To maximise the sensitivity, $H \rightarrow c \bar{c}$ decays are thus searched for by ATLAS only for Higgs bosons produced in association with a $Z$ boson decaying to $e e$ or $\mu \mu$. This leads to a $\sigma \times B R$ which is just $3 \times 10^{-5}$ of the total Higgs boson production cross section at $13 \mathrm{TeV}$.

The search has been performed on $36 \mathrm{fb}^{-1}$ of $13 \mathrm{TeV} p p$ collisions [5]. Higgs boson candidates are identified as pairs of jets, at least one of which passes $c$-tagging, a multivariate algorithm for $c$ jet identification based on multiple properties of charged particle tracks such as impact parameters and the presence of secondary vertices. An excess above the expected background is searched for around $125 \mathrm{GeV}$ in the distribution of the invariant mass of the two jets from the decay of the Higgs boson candidate, in four event categories based on the number of $c$-tagged jets and the $Z$ boson transverse momentum. No significant excess is found (see Figure 1(b) for an example), and a 95\% CL upper limit on $\sigma \times B R$ is set to be 110 times the SM prediction.

\section{Searches for lepton-flavour violating $H \rightarrow \ell \tau$ decays}

Lepton-flavour violating decays $H \rightarrow \ell \tau$ in the SM can only proceed through neutrino mixing and are thus expected to have vanishingly small BR. Theories with a larger Higgs sector, including non-diagonal couplings $y_{\ell \tau}$, can enhance such BR.

A search for $H \rightarrow \ell \tau$ decays has been performed by ATLAS with $36 \mathrm{fb}^{-1}$ of $13 \mathrm{TeV} p p$ collisions [6]. Opposite-sign $\ell \tau$ pairs are selected, where the $\tau$ candidate is reconstructed either through its leptonic decays $\tau \rightarrow \ell^{\prime}, \ell^{\prime} \neq \ell\left(\tau_{1^{\prime}}\right)$, or its hadronic 1- or 3-prong decays $\left(\tau_{\text {had }}\right)$. For each of the two $\ell \tau$ final states, selected events are assigned to one of either (i) four signal regions (SRs), based on the $\tau$ lepton decay type and whether events have a signature consistent with Higgs boson production in the vector-boson-fusion (VBF) mode, or (ii) four control regions (CRs) enriched in top or $Z \rightarrow \tau \tau$ events, which constitute the main background processes. The signal and background yields are determined by a simultaneous fit to the signal and control regions. The fit uses as inputs 


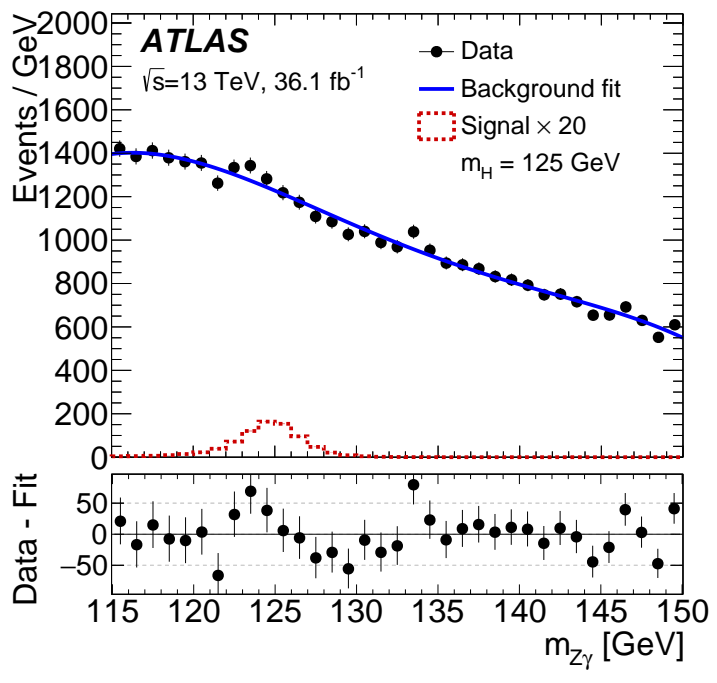

(a)

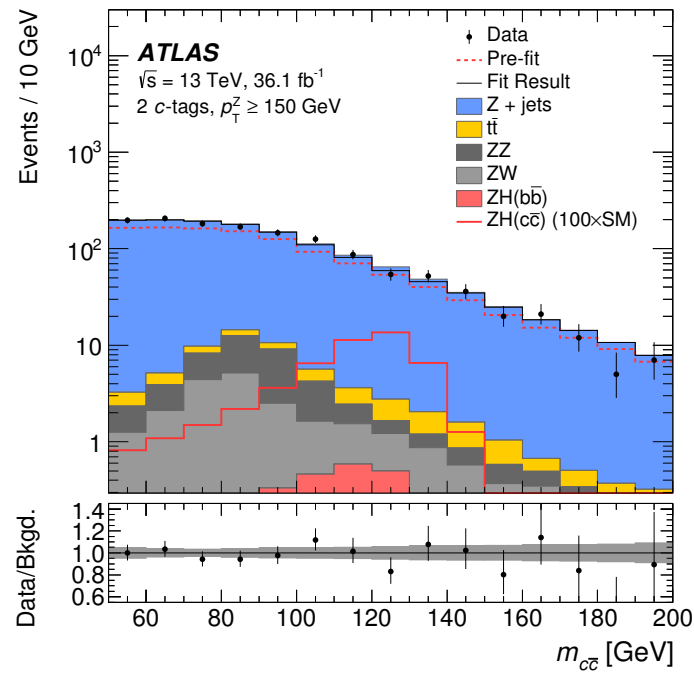

(b)

Figure 1: (a) The invariant $Z \gamma$ mass $\left(m_{Z \gamma}\right)$ distribution of events satisfying the $H \rightarrow Z \gamma$ selection combining all event categories. [2] The points represent the data and the statistical uncertainty. The solid line is the sum of the background-only fits to the data performed in the individual categories. The dashed histogram corresponds to the expected signal for a SM Higgs boson with $m_{H}=125 \mathrm{GeV}$ decaying to $Z \gamma$ with a rate 20 times the SM prediction. The bottom part of the figure shows the residuals of the data with respect to the background-only fit. (b) Observed and predicted $m_{c \bar{c}}$ distribution for events with two $c$-tagged jets and reconstructed $Z$ boson transverse momentum above $150 \mathrm{GeV}$ [5]. The expected signal is scaled by a factor of 100. The expected backgrounds after the fit to the data are shown as the solid histograms. The predicted background from the simulation is shown as red dashed histograms. The ratios of the data to the fitted background are shown in the lower panels. The error bands indicate the sum in quadrature of the statistical and systematic uncertainties in the background prediction.

the event yields in the four CRs and the distributions of the score of a boosted decision tree (BDT) for the events in the four SRs. The BDT is computed from several kinematic quantities characterising the particles in the final state. No significant signal is observed in any category (see Figure 2(a) for an example). The best-fit value of the BR is consistent with 0 within $1 \sigma$ in both final states. The 95\% CL upper limits on the branching ratios to $\ell \tau$ are $0.47 \%(H \rightarrow e \tau)$ and $0.28 \%(H \rightarrow \mu \tau)$. The results are limited by the systematic uncertainties, among which the main ones are related to the jet reconstruction and selection performance and to the estimate of background from mis-identified jets and leptons.

\section{Searches for $H \rightarrow \mu \mu$ decays}

Higgs boson decays to muon pairs are induced by the $y_{\mu}$ Yukawa coupling and, due to the smallness of the muon mass, are expected in the SM to have a BR of only about $2 \times 10^{-4}$.

A search for $H \rightarrow \mu \mu$ decays has been performed on the full $13 \mathrm{TeV} p p$ collision ATLAS data [7], corresponding to an integrated luminosity of $139 \mathrm{fb}^{-1}$. The inclusion of data collected 
in 2018 with respect to the previous released $H \rightarrow \mu \mu$ search, together with the refinement of the techniques employed by the analysis, has led to a 50\% improvement of its sensitivity.

Events are required to contain two opposite-sign muons with transverse momenta $\left(p_{\mathrm{T}}\right)$ above 27 and $15 \mathrm{GeV}$ and invariant mass in the region 110-160 GeV. The selection has an expected signal efficiency of $60 \%$, leading to a non negligible expected signal yield of 860 events. Unfortunately, the cross section for dimuon Drell-Yan production is much larger, leading to an expected number of background events after the selection of 2.5 millions, of which about one fifth has invariant mass in the $120-130 \mathrm{GeV}$ window close to the Higgs boson mass, where the signal is expected to peak.

After the inclusive selection, the sensitivity of the analysis is increased by splitting events in twelve categories with different $S / B$, based on the number of jets in the event and on the scores of 4 BDT discriminants using as inputs the kinematic distributions of the dimuons, of up to two highest- $p_{\mathrm{T}}$ jets, and of $E_{\mathrm{T}}^{\mathrm{miss}}$. The signal and background yields are determined by a fit to the dimuon invariant mass. The signal resolution, close to $3 \mathrm{GeV}$, is improved by $3 \%$ by taking into account final state radiation. The analytical model used to parametrise the background shape in the fit is determined from studies on large simulated samples of Drell-Yan events, requiring the chosen model to give a good description of the simulation and of the data (outside the region 120-130 $\mathrm{GeV}$ ) and to lead to a bias on the signal that is less than $20 \%$ of its statistical uncertainty. The parameters of the background model are determined from the fit to the data, simultaneously with the yields. The expected significance of a signal with the SM rate is $1.5 \sigma$.

In data only a small, statistically insignificant excess over the expected background is observed (Figure 2(b)), with a significance of $0.8 \sigma$, corresponding to $\sigma \times B R=0.5 \pm 0.7$ times the SM prediction. A 95\% CL upper limit on $\sigma \times B R$ of 1.7 times the SM prediction is set. The results are limited by the statistical uncertainties induced by the fluctuations of the background.

\section{Conclusion}

The results of the searches for several rare or forbidden Higgs boson decays have been presented: (i) $H \rightarrow Z \gamma$, (ii) $H \rightarrow M \gamma, M=\left(q \bar{q}\right.$ ), (iii) $Z H, H \rightarrow c \bar{c}$, (iv) $H \rightarrow e \tau$ and $\mu \tau$, (v) $H \rightarrow \mu^{+} \mu^{-}$. These searches are potentially sensitive to BSM physics affecting the Yukawa couplings to the fermions of the first two generations or leading to non-flavour-diagonal couplings. The searches have been performed on $13 \mathrm{TeV} p p$ collision data recorded by the ATLAS detector at the LHC. No significant signal was observed. For all final states but the dimuon one, three times more data already recorded by ATLAS before the two-year long shutdown started at the beginning of 2019 are available for analysis. For the $H \rightarrow \mu \mu$ decay, the full dataset collected so far was used, and the sensitivity of the analysis $(1.5 \sigma)$ is getting closer to that needed to observe this decay if the branching ratio is SM-like, for which data collected in the next LHC data taking run will be crucial.

\section{References}

[1] ATLAS Collaboration, “The ATLAS Experiment at the CERN Large Hadron Collider," JINST 3 (2008) S08003.

[2] ATLAS Collaboration, "Searches for the $Z \gamma$ decay mode of the Higgs boson and for new high-mass resonances in $p p$ collisions at $\sqrt{s}=13 \mathrm{TeV}$ with the ATLAS detector," JHEP 1710 (2017) 112. 


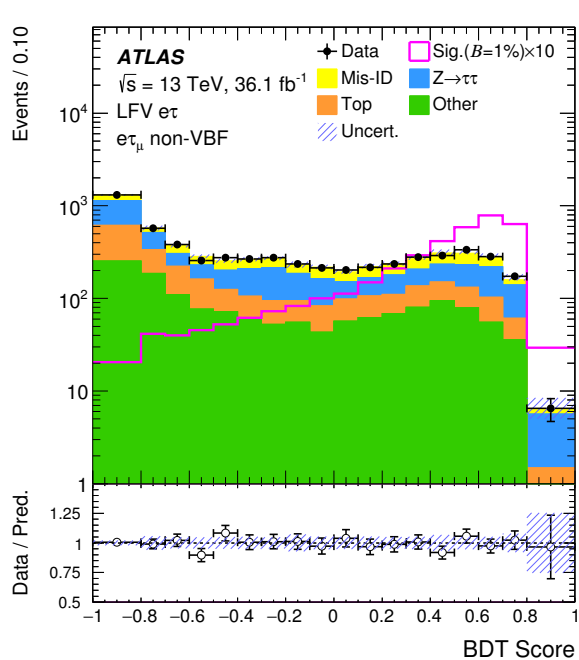

(a)

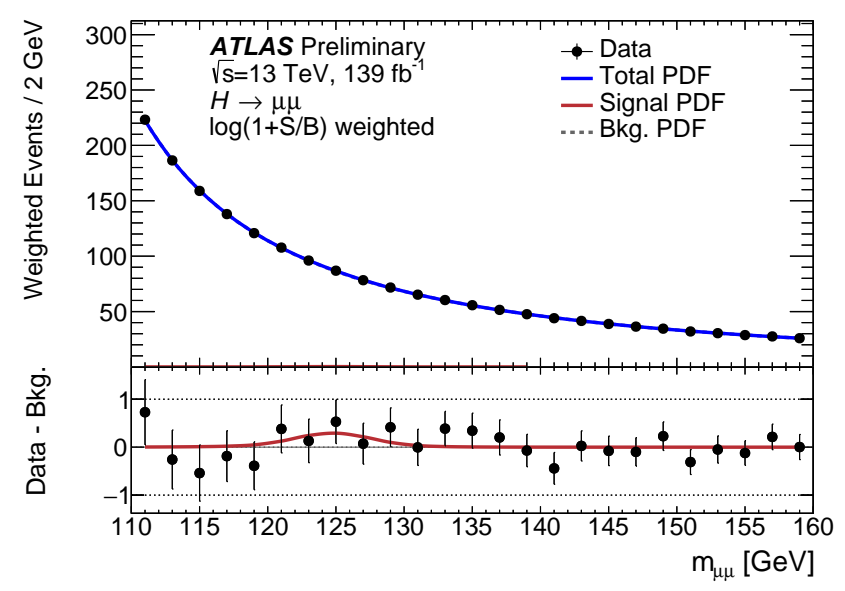

(b)

Figure 2: (a) Distribution of the BDT score after the background+signal fit in the $e \tau_{\mu}$ non-VBF signal region of the $H \rightarrow e \tau$ search, with the LFV signal overlaid, normalized with $B R(H \rightarrow e \tau)=1 \%$ and enhanced by a factor 10 for visibility [6]. The size of the combined statistical, experimental and theoretical uncertainties of the background is indicated by the hatched bands. (b) Dimuon invariant mass spectrum in all the $H \rightarrow \mu \mu$ analysis categories observed in data (dots with error bars) [7]. The signal, background and total probability density functions (PDF) are also shown (lines).

[3] ATLAS Collaboration, "Search for exclusive Higgs and $Z$ boson decays to $\phi \gamma$ and $\rho \gamma$ with the ATLAS detector," JHEP 1807 (2018) 127.

[4] ATLAS Collaboration, "Searches for exclusive Higgs and $Z$ boson decays into $J / \psi \gamma, \psi(2 S) \gamma$, and $\Upsilon(n S) \gamma$ at $\sqrt{s}=13 \mathrm{TeV}$ with the ATLAS detector," Phys. Lett. B 786 (2018) 134.

[5] ATLAS Collaboration, "Search for the Decay of the Higgs Boson to Charm Quarks with the ATLAS Experiment,” Phys. Rev. Lett. 120 (2018) 211802.

[6] ATLAS Collaboration, "Searches for lepton-flavour-violating decays of the Higgs boson in $\sqrt{s}=13$ TeV pp collisions with the ATLAS detector," arXiv:1907.06131 [hep-ex].

[7] ATLAS Collaboration, "A search for the dimuon decay of the Standard Model Higgs boson in $p p$ collisions at $\sqrt{s}=13 \mathrm{TeV}$ with the ATLAS Detector," ATLAS-CONF-2019-028, http://cds.cern.ch/record/2682155. 